\title{
Influence of the sample anticoagulant on the measurements of impedance aggregometry in cardiac surgery
}

\author{
Cristina Solomon' \\ Michael Winterhalter' \\ Isabel Gilde' \\ Ludwig Hoy ${ }^{2}$ \\ Andreas Calatzis ${ }^{3}$ \\ Niels Rahe-Meyer' \\ 'Department of Anesthesiology, \\ Hannover Medical School, Hannover, \\ Germany; ${ }^{2}$ Institute for Biometry, \\ Hannover Medical School, Hannover, \\ Germany; ${ }^{3}$ Department \\ of Hemostasis Transfusion Medicine, \\ University Hospital \\ Munich, Munich, Germany
}

\begin{abstract}
Background: The standard method of assessment of platelet function is represented by light transmission aggregometry (LTA), performed in citrated platelet-rich plasma (PRP). With LTA, decrease and subsequent post-cardiopulmonary bypass (CPB) recovery of platelet function have been reported during cardiac surgery. Multiple electrode aggregometry (MEA) may be used as point-of-care method to monitor perioperative changes in platelet function. Since MEA assesses macroaggregation which is influenced by the plasmatic levels of unbound calcium, citrate may be inadequate as anticoagulant for MEA. We used citrate and heparin for MEA samples, to see with which anticoagulant the intraoperative decrease and postoperative recovery in platelet function previously described with other aggregometric methods in cardiac surgery may be observed with MEA.
\end{abstract}

Methods: Blood was obtained from 60 patients undergoing routine cardiac surgery and the samples were collected in standard tubes containing unfractionated heparin $(50 \mathrm{U} / \mathrm{mL})$ or trisodium citrate (3.2\%). The samples were obtained before $\mathrm{CPB}$, at 30 minutes on $\mathrm{CPB}$, end of $\mathrm{CPB}$ and on the first postoperative day. MEA was performed using the Multiplate ${ }^{\circledR}$ analyzer. Collagen (COLtest, $100 \mu \mathrm{g} / \mathrm{mL}$ ) and TRAP-6 (thrombin receptor activating peptide, TRAPtest, $1 \mathrm{mM} / \mathrm{mL}$ ) were used as aggregation agonists.

Results: Platelet aggregometric response decreased significantly during CPB. Platelet aggregation assessed using TRAP-6 as agonist on heparinized blood significantly correlated with the duration of CPB $(r=-0.41, p=0.001,2$-tailed Pearson test). The aggregometric analysis performed on the first postoperative day showed a significant recovery in platelet activity in the samples containing heparin (increase from $30 \pm 22 \mathrm{U}$ to $46 \pm 27 \mathrm{U}$ for the COLtest and from $70 \pm 34 \mathrm{U}$ to $95 \pm 32 \mathrm{U}$ for the TRAPtest, $\mathrm{p}<0.001$, Student's t-test), while no significant recovery of platelet function could be established in the MEA measurements performed with citrated blood.

Conclusions: The choice of blood sample anticoagulant used for impedance aggregometry influenced the platelet aggregation response. Postoperative platelet function recovery was only detected in the heparinized samples. Heparin seems to be better suited than citrate for the analysis of impedance aggregometry in heart surgery.

Keywords: cardiac surgery, impedance aggregometry, platelet recovery, sample anticoagulant

\section{Introduction}

Platelet dysfunction occurring during cardiac surgery with cardiopulmonary bypass (CPB) contributes to perioperative excessive bleeding (Hartmann et al 1992). Several aggregometry methods have been proposed for the assessment of platelet function during and after cardiac surgery. Using light transmission aggregometry (LTA) assessed in platelet rich plasma, decrease and subsequent post-CPB recovery of platelet function have been reported during cardiac surgery (Boldt et al 1994). Whole blood aggregometry (WBA) introduced in 1980 by Cardinal examines platelet aggregation by 
measuring changes in impedance between two electric wires after platelet agonist addition to the blood sample (Cardinal and Flower 1980). Multiple electrode aggregometry (MEA), a new application of whole blood aggregometry (von Pape et al 2007; Seyfert et al 2007; Mengistu et al 2008), uses the same principle and may be of use as point-of-care method for the monitoring of perioperative changes in platelet function.

The choice of anticoagulant for MEA analysis is still unclear. Based on previous experience with LTA, in many studies of WBA sodium citrate has been used as anticoagulant for the blood samples, because of its ability to bind calcium and block the onset of coagulation. Inhibition of platelet function by the chelation of unbound calcium by citrate has been reported (Bretschneider et al 1994; Wallen et al 1997) and one strategy to circumvent calcium chelation is the use of recombinant hirudin or D-phenylalanyl-L-prolyl-L-arginine chloromethyl ketone (PPACK) as anticoagulant (Glusa and Markwardt 1990; Wallen et al 1997). However, since no blood collection tubes containing these anticoagulants are commercially available for routine testing in the perioperative setting, standard heparin tubes may represent an alternative for MEA analyses. The use of heparin as blood sample anticoagulant for the measurements of platelet function in LTA has been discouraged (Wallen et al 1997), because heparin increases in vitro formation of microaggregates (less than 20 platelets per aggregate) which cause opacity of the PRP sample and inaccurate high test results in LTA. In WBA, however, the formation of macroaggregates $(>100$ platelets per aggregate) is not significantly affected by ex vivo low dose heparinisation (Belcher et al 2000).

We compared the use of citrate and heparin as anticoagulants for MEA in cardiac surgery, to see with which of the two anticoagulants the intraoperative decrease and postoperative recovery in platelet function previously described with other aggregometric methods in cardiac surgery may be observed with MEA.

\section{Methods}

Following local Ethics Committee approval, 60 consecutive patients who were scheduled for elective cardiac surgery were enrolled in this prospective study. After giving informed written consent, the patients were included consecutively by one anesthetic group of the study centre. Exclusion criteria consisted of any known congenital or acquired bleeding disorders, severe liver disease, age under 18 years, pregnancy or nursing and emergency operation. Before the operation, the patients were interviewed on intake of aspirin, nonsteroidal antiinflammatory drugs, glycoprotein (GP) IIb-IIIa antagonists, thienopyridines or antibiotics known to influence platelet aggregation.

The operation and anesthetic management was similar in all patients. Induction of anaesthesia was done with etomidate, fentanyl, and pancuronium and maintained with sevoflurane and propofol. Analgesia was obtained by administering repetitive boluses of fentanyl. During induction of anaesthesia all patients received $500 \mathrm{ml}$ of lactated Ringer's solution and $500 \mathrm{ml}$ of hydroxyethyl starch $(6 \%$ HES 200/0.5). A bolus of pig mucosal heparin $400 \mathrm{IU} / \mathrm{kg}$ was administered before institution of CPB and 2 million kallikrein-inhibiting units (KIU) of aprotinin were given on CPB. After the initial anticoagulation, additional doses of heparin were given to maintain activated clotting time (ACT) above $480 \mathrm{~s}$. After CPB, heparin was neutralized with protamine sulfate, $1 \mathrm{mg}$ protamine/100 $\mathrm{U}$ of total heparin dose and the reversal of the heparin effect was controlled by ACT less than 150 s.

Blood samples were drawn before the beginning of CPB, 30 min on CPB, at the end of CPB, and on the first postoperative day from a radial artery catheter (20 Gauge) into commercially available Monovette ${ }^{\circledR}$ collection tubes (Sarstedt, Nuembrecht, Germany) which contained heparin $(50 \mathrm{U} / \mathrm{mL})$ or citrate $(3.2 \%)$ as anticoagulant.

Multiple electrode aggregometry was performed using the Multiplate $^{\circledR}$ analyzer (Dynabyte medical, Munich, Germany). Detailed description of the method is available in the literature (Tóth et al 2006; Seyfert et al 2007). Briefly, $300 \mu \mathrm{l}$ of saline and $300 \mu \mathrm{l}$ of either heparinized or citrated blood were pipetted into a test cell and stirred using a magnetic stirrer. After an incubation period of 3 minutes at $37{ }^{\circ} \mathrm{C}$, the agonist was added and the recording started. During the following 6 minutes the ability of platelets to adhere and aggregate onto 4 metal wires, which form 2 independent sensor units was measured by the electrical resistance change between the sensor wires. The impedance change caused by the adhesion of the platelets onto the sensor surfaces was plotted against time and the area under the aggregation curve was used to measure the aggregation response, quantified in arbitrary units (U). Since the change in impedance was measured simultaneously on 2 sensor units, the results of each test represented the mean value of the 2 aggregation curves obtained. However, if the two aggregation curves obtained were too different (correlation coefficient $r<0.99$, calculated by the analyzer), the measurement was marked automatically as incorrect and had to be repeated. The following agonists were used for the analysis (test name and final concentration 
in the parentheses): collagen (COLtest, $100 \mu \mathrm{g} / \mathrm{mL}$ ) and TRAP-6 (thrombin receptor activating peptide, TRAPtest, $1 \mathrm{mM} / \mathrm{mL}$ ). The aggregation tests were performed in close proximity to the operation theater. The instrument and all reagents are commercially available from the manufacturer of the device.

\section{Statistical analysis}

Data is presented as mean $\pm \mathrm{SD}$. For comparisons between consecutive time points, the closed testing procedure was applied to test the null hypotheses of equal mean values at the family-wise error rate of $\alpha=0.05$. Hotelling's $\mathrm{T}^{2}$ test was used for the joint hypotheses and the paired Student's t-test for the component hypotheses. The normality of the differences was assessed by use of the Kolmogorov-Smirnov test. Correction for multiple comparisons was performed with the Bonferroni-Holm method. The correlation between CPB duration, platelet count, hematocrit and platelet aggregation was assessed with the 2-tailed Pearson test. Bland Altman plots were generated to compare COLtest and TRAPtest between citrated and heparin blood. A linear mixed model with the difference of two measurements as dependent, the average as covariate, the time point including interaction to the average as fixed factors and the patient as random factor was applied to analyse the bias. Data analysis was performed with Statview 9.1 (SAS Institute, Cary, NC), SPSS 14.0 (SPSS Inc., Chicago, IL), and Microsoft Excel (Microsoft Corp., Redmond, WA).

\section{Results}

Table 1 summarizes the demographic data of the 60 patients included in the study, as well as the parameters of the operation management.

The perioperative changes in platelet aggregometry are presented in Figure 1.

For the COLtest performed in citrated blood, the baseline aggregation response measured by MEA was $28 \pm 14 \mathrm{U}$. After 30 minutes on $\mathrm{CPB}$, the response to collagen was significantly reduced to $22 \pm 14 \mathrm{U}$ ( $\mathrm{p}<0.001$, Student's t-test). At the end of the CPB, the response to collagen had reduced further to $17 \pm 12 \mathrm{U}(\mathrm{p}=0.01$, Student's t-test). One day post-CPB, the response to collagen in citrated blood $(18 \pm 11 \mathrm{U})$ was below the preoperative level and not significantly different from the response at the end of the CPB ( $\mathrm{p}=0.7$, Student's t-test).

For the COLtest in heparinized blood, the preoperative response to collagen was $70 \pm 23 \mathrm{U}$. At 30 minutes on $\mathrm{CPB}$, the response to collagen in heparin containing blood
Table I Demographic and operation data

\begin{tabular}{ll}
\hline Parameter & $\begin{array}{l}\text { mean } \pm \text { SD or } \mathbf{~} \\
\text { (percentage) }\end{array}$ \\
\hline Age (years) & $63.6 \pm 15.4$ \\
Sex (male) & $42(70 \%)$ \\
Aspirin medication & $28(46.7 \%)$ \\
Not discontinued for more & $5(8.3 \%)$ \\
than 4 days & \\
Clopidogrel medication & $5(8.3 \%)$ \\
Not discontinued for more & $2(3.3 \%)$ \\
than 7 days & \\
Gpllb/llla antagonist not & $1(1.7 \%)$ \\
discontinued for more & \\
than 7 days & \\
$\begin{array}{l}\text { Deepest cooling } \\
\text { temperature }\end{array}$ & $32.5 \pm 2.7$ \\
$\begin{array}{l}\text { Temperature at the end } \\
\text { of CPB }\end{array}$ & $36.6 \pm 0.2$ \\
Operation time (minutes) & \\
CPB time (minutes) & $223.7 \pm 77.3$ \\
Aortic cross-clamp time & $119.7 \pm 60.5$ \\
(minutes) & $66.5 \pm 39.8$ \\
\hline
\end{tabular}

Abbreviations: $\mathrm{SD}$, standard deviation; $\mathrm{N}$, number of patients; $\mathrm{CPB}$, cardiopulmonary bypass.

had decreased significantly from the preoperative value to $35 \pm 22 \mathrm{U}(\mathrm{p}<0.001$, Student's t-test). At the end of CPB, the response to collagen was $30 \pm 22 \mathrm{U}$, not significantly different from the previous time point ( $p=0.06$, Student's t-test). Unlike the aggregometry analysis in citrated blood, on the first postoperative day a significant improvement in platelet response to collagen compared to the previous day was noted in heparinized blood ( $46 \pm 27 \mathrm{U}$ compared to $30 \pm 22 \mathrm{U} ; \mathrm{p}<0.001$; Student's t-test).

For the TRAPtest performed in citrated blood, the platelet aggregation response before CPB was $54 \pm 18 \mathrm{U}$. This response decrease significantly to $44 \pm 22 \mathrm{U}$ after $30 \mathrm{~min}$ CPB time ( $\mathrm{p}<0.001$; Student's t-test), and showed a further decrease to $32 \pm 17 \mathrm{U}$ at the end of CPB ( $p<0.001$; Student's t-test). On the first postoperative day, the response to TRAP-6 was significantly lower than at the end of CPB, $27 \pm 14 \mathrm{U}$ compared to $32 \pm 17 \mathrm{U}(\mathrm{p}=0.015$; Student's t-test).

For the TRAPtest performed in blood anticoagulated with heparin, the pre-CPB aggregometric response was $105 \pm 21 \mathrm{U}$. After 30 minutes on CPB the aggregometric response decreased to $83 \pm 36 \mathrm{U}$ ( $\mathrm{p}<0.001$; Student's t-test) and showed a further significant decrease at the end of CPB to $70 \pm 34 \mathrm{U}(\mathrm{p}=0.002$; Student's t-test). Unlike the citrated blood sample results, the response to TRAPtest in 


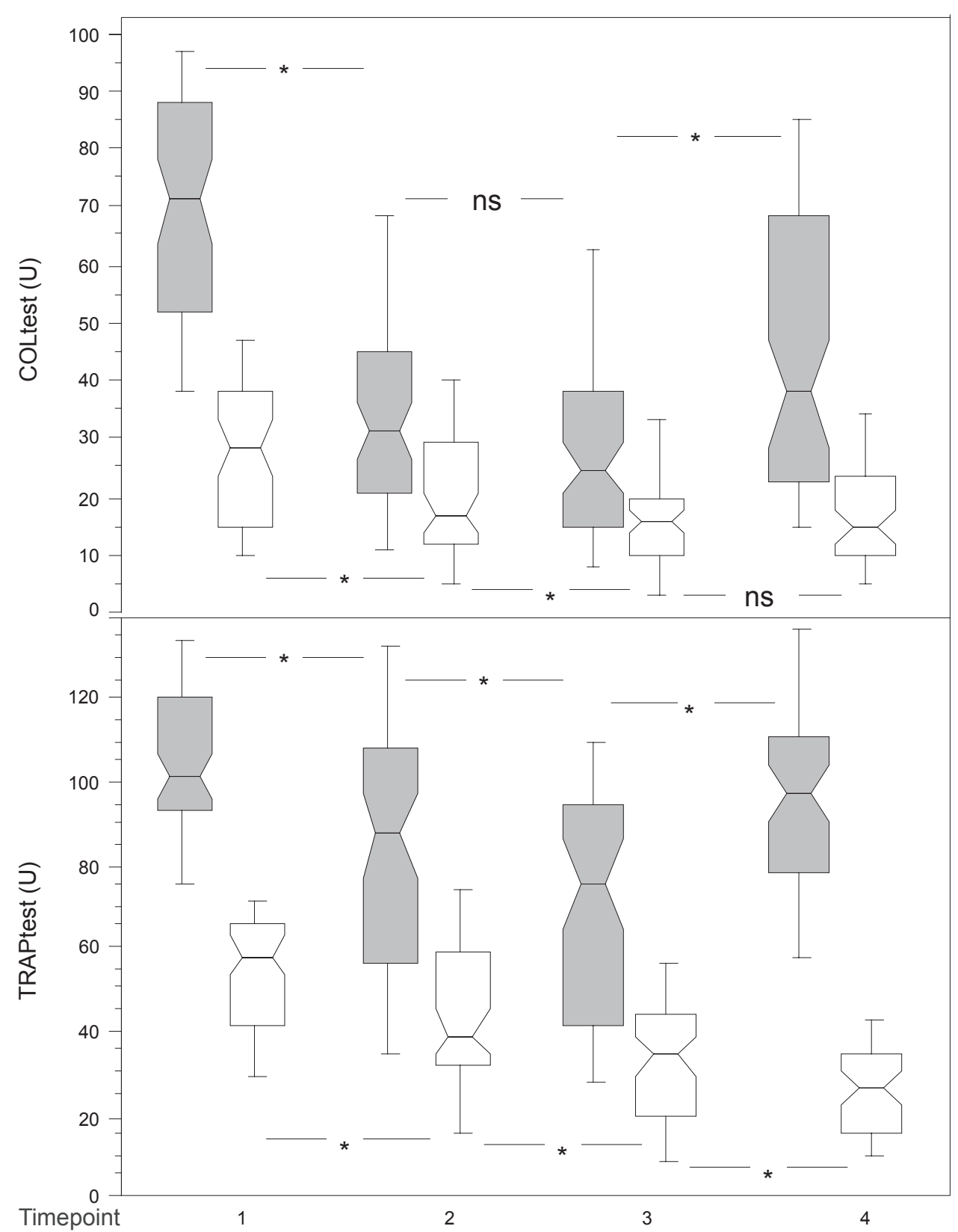

Figure I Time course of aggregation results. Data are presented as Box-and-Whisker plots showing mean, 5\%, 25\%, 75\%, and 95\% percentiles. Grey box plots represent the results of the analysis of heparin blood, white box plots of citrated blood. $n=60$ patients; time point ( 1 ) before the beginning of cardiopulmonary bypass (CPB); (2) at 30 minutes on CPB; (3) at the end of CPB; (4) on the first postoperative day. There was a significant difference of the results of citrated vs heparin blood for all time points and tests Notes: ${ }^{*} \mathrm{p}<0.05$ (closed testing procedure; Hotelling's $\mathrm{T}^{2}$ test). The most important difference between the analyses of citrated and heparin blood is that an increase in platelet function is detected between the end of operation and the first postoperative day only in heparin blood but not in citrated blood.

Abbreviations: CPB, cardiopulmonary bypass; ns, not significant.

the heparinized blood on the first postoperative day showed a significant recovery of the platelet aggregometric response compared to the end of $\mathrm{CPB}$ values $(95 \pm 32 \mathrm{U}$ compared to $70 \pm 34 \mathrm{U}, \mathrm{p}<0.001$; Student's t-test). The differences between tests remained significant after correction for multiple tests.

The difference between the impedance aggregometry in heparin blood minus citrated blood was positively correlated to the average of the two measurements ( $p<0.001$ for COLtest and TRAPtest). Both intercept and regression coefficients were different between the time points $(p<0.05$ in both tests on the linear mixed model analysis).

The aggregometry analyses were performed at mean platelet count of $239 \pm 56 * 10^{9} / \mathrm{L}$ before the beginning of the $\mathrm{CPB}$. At the end of $\mathrm{CPB}$ platelet count decreased to $123 \pm 45 * 10^{9} / \mathrm{L}$. On the first postoperative date, the 
MEA analyses were performed at a platelet count of $134 \pm 34 * 10^{9} / \mathrm{L}$. Except for the significant correlation between platelet count and aggregometry in COLtest at the end of bypass ( $\mathrm{r}=0.34 ; \mathrm{p}=0.007 ; 2$-tailed Pearson test), no other correlation were observed between platelet count and aggregometry with any of the two agonists, collagen and TRAP-6, for the remaining time points. Hematocrit values were $40 \pm 4 \%$ before $\mathrm{CPB}, 29 \pm 3 \%$ at the end of CPB and $31 \pm 3 \%$ on the first postoperative day. No correlations were observed with the 2-tailed Pearson test between hematocrit and aggregometry at any time point.

Regarding the total CPB time, the results of the COLtest performed in either citrated or heparinized blood did not correlate significantly with the duration of CPB $(\mathrm{r}=-2.41$, $\mathrm{p}=0.06$ and $\mathrm{r}=-2.81, \mathrm{p}=0.3$, respectively; 2-tailed Pearson test). Also for the TRAPtest performed in citrated blood no correlation was found with the total CPB time ( $\mathrm{r}=-2.45, \mathrm{p}=0.059,2$-tailed Pearson test). Only TRAPtest performed in heparinized blood correlated significantly with CPB time ( $\mathrm{r}=-0.41 ; \mathrm{p}=0.001 ; 2$-tailed Pearson test).

\section{Discussion}

The main finding of our study is that the MEA analyses performed in heparinized blood, unlike those in citrated blood, reflect intraoperative decrease and postoperative recovery of platelet aggregation. Moreover, aggregometry induced by using TRAP-6 in heparinized blood correlated with the duration of the $\mathrm{CPB}$. Based on our results, heparin seems to be better suited than citrate as anticoagulant for impedance aggregometry performed as point-of-care method to assess platelet function during cardiac surgery.

\section{Assessment of platelet function in heparin blood}

The gradual change observed in the aggregometric response of the using heparinized blood samples during cardiac surgery is in accordance to the response described by other studies using either LTA or WBA in this setting (Boldt et al 1993, 1994; Slaughter et al 2001; Poston et al 2005; Mengistu et al 2008). CPB procedures resulted in a pronounced reduction in platelet aggregation response with both collagen (COLtest) and thrombin receptor activator (TRAPtest) as activator. 30 minutes after beginning of $\mathrm{CPB}$, reduction in platelet response and function was more pronounced in COLtest, as collagen is known to be a weaker platelet stimulus than the thrombin receptor activator TRAP-6, which binds to the thrombin receptors on the platelet surface and mimics the strong stimulatory action mediated by thrombin
(Landesberg et al 2005). From this time point to the end of $\mathrm{CPB}$, an additional significant reduction in platelet response was recorded in TRAPtest but not in COLtest, as well as a time dependency of aggregometry performed with TRAPtest. This may be explained by the fact that the response to collagen is already maximally inhibited as soon as 30 minutes after the start of CPB. On the first postoperative day a significant increase in the aggregometric response was observed in both COLtest and TRAPtest. In the latter, the aggregation values were comparable to pre-CPB values.

Heparin in high doses is known to have an activating effect on platelets, explored both ex vivo and clinically, during cardiac surgery (Chen et al 1991; Belcher et al 2000). The in vivo effect of heparin seen during CPB seems to be rather due to plasmatic changes induced by heparin then to the direct effect of heparin on platelets (Kestin et al 1993; Muriithi et al 2000). The use of heparin or low molecular heparins as anticoagulant is precluded in LTA, due to the formation of platelet microaggregates that impede the measurement (Born and Cross 1963; Kingston et al 2001). This observation led to citrated blood being recommended as anticoagulant for WBA, a recommendation that was extended also to the newer MEA. The heparin-induced formation of platelet microaggregates, however, seems to be less influencing of MEA, since MEA does not assess free microaggregates but only macroaggregates that bind firmly onto the electrodes. Using heparin as sample anticoagulant in MEA allows the assessment of the platelet function during the whole time course of the operation. Thus the platelet function of a patient still on CPB and under full heparinization may be measured in order to predict the coagulation therapy necessary at the end of CPB and to assess the efficacy of platelet concentrates transfusion.

\section{Assessment of platelet function in citrated blood}

The aggregometric response of platelets in citrated blood was constantly lower than in heparinized blood, for both COLtest and TRAPtest (Figure 1). The Bland Altman plots (Figure 2a-b) for the COLtest and TRAPtest showed that the response of platelets in heparinized blood was consistently higher than the same measurement in citrated blood, but with different slopes between tests and between time points. This finding is consistent to those of other studies showing lower aggregometric response of platelets to collagen in citrated blood than with other anticoagulants (Wallen et al 1997). After 30 minutes of CPB, a mean decrease in collageninduced aggregation of $22 \%$ compared to the pre-CPB 
A

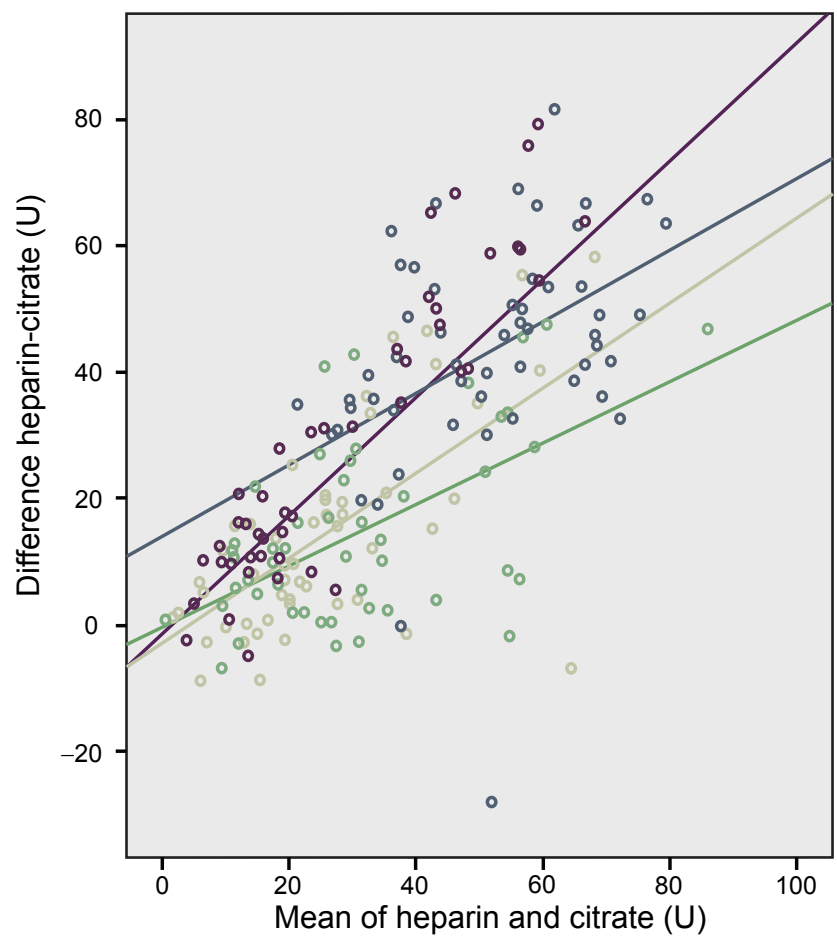

B

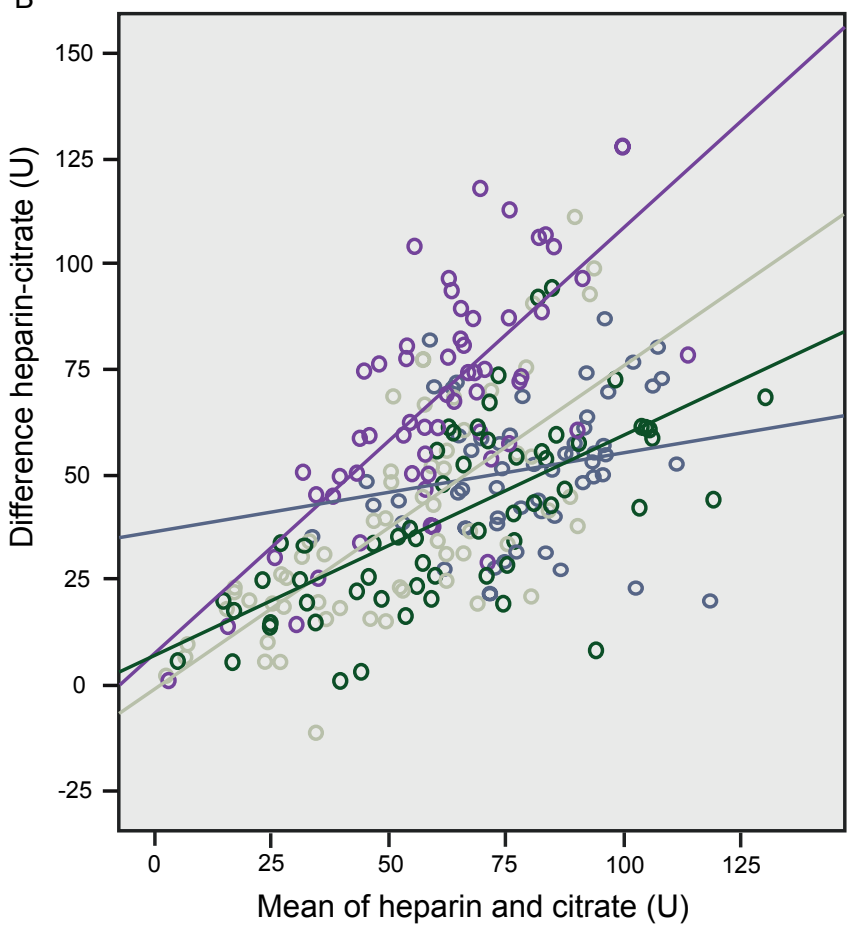

Time point

? 1

2
+4

$\mathrm{R}$ Sq Linear $=0,681$

$\mathrm{R}$ Sq Linear $=0,451$

$\mathrm{R}$ Sq Linear $=0,343$

$\mathrm{R}$ Sq Linear $=0,261$

$$
\begin{gathered}
\text { Time point } \\
\text { a } 1 \\
2 \\
\text { a } 3 \\
\text { a } 4
\end{gathered}
$$

R Sq Linear $=0,523$

$\mathrm{R}$ Sq Linear $=0,519$

R Sq Linear $=0,423$

$\mathrm{R}$ Sq Linear $=0,043$

Figure 2 Bland-Altman plot for COLtest (A) and TRAPtest (B) performed in blood anticoagulated with heparin or citrate, respectively. $\mathrm{n}=60$ patients; time point (I) before the beginning of cardiopulmonary bypass (CPB); (2) at 30 minutes on CPB; (3) at the end of CPB; (4) on the first postoperative day. Particularly for TRAPtest, some samples showed an excellent aggregation in heparin blood while almost no aggregation in citrated blood was observed. 
response observed in citrated blood, whereas the decrease was of $50 \%$ between the same time points in heparinized blood. Our results are in agreement with other reports from the literature, where reduction during CPB in the platelets' answer to stimulation with collagen has been described for both LTA and WBA (Boldt et al 1993, 1994; Slaughter et al 2001; Poston et al 2005; Mengistu et al 2008). An important finding of our study is the difference between the aggregometric measurements performed with the two types of anticoagulant on the first postoperative day. A significant increase in the aggregometric response in heparinized blood compared to the previous time point, the end of CPB, was noted. In citrated blood, aggregometric results of the first postoperative day did not differ significantly from the results at the end of CPB. Postoperative recovery of platelet function has been investigated in literature, mostly by means of LTA performed in citrated blood, which showed increased platelet activity as early as 5 hours after surgery (Boldt et al 1993, 1994). In WBA performed with citrated blood 3.8\% using higher dose of collagen $(5 \mu \mathrm{g} / \mathrm{mL})$ moderate recovery of platelet function after off pump cardiac surgery was noted (Poston et al 2005). So far, there are no reports on postCPB recovery of platelet activity assessed in whole blood aggregometry activated with TRAP-6.

Citrate diminishes the extracellular calcium concentration, with severe effects on platelet aggregation (Born and Cross 1963). With low citrate concentration, the concentration of calcium remains high enough to allow minimal thrombin generation that induces platelet stimulation. If the citrate concentration is high and the extracellular calcium concentration is low for a certain period of time - the manufacturer of the MEA recommends that the blood samples should not be analyzed immediately but after 30 minutes at the earliest - intracellular calcium concentration decreases. With a low intra-and a low extracellular level of calcium enhanced thromboxane generation occurs (Wallen et al 1997), inducing falsely increased aggregation response in the citrated blood samples. A normal calcium level would have presumably resulted in even lower perioperative aggregometric results, but the correction of this limitation is not possible without affecting coagulation in the whole blood citrated samples used for MEA.

As for the anesthetic management, platelet function is also known to be influenced by the heparin dosage, the heparin neutralization at the end of CPB and by the administration of aprotinin. The effect of heparin on macroaggregation appears early during CPB and is significant with doses as low as $30 \mathrm{U} / \mathrm{kg}$, while addition of up to $270 \mathrm{U} / \mathrm{kg}$ induces no significant additional impairment of platelet function (Muriithi et al 2000). Since all patients included in the study received high dosage heparin during bypass, differences between individual doses were not assessed with respect to the MEA measurements. All patients also received aprotinin in low dose during CPB $\left(2 * 10^{6} \mathrm{KIU}\right)$, a dose shown to have a protective effect on platelet aggregation, as assessed in electron microscopy measurements by Lavee and colleagues (1993). With our study design, however, the effect of aprotinin could not be isolated.

\section{Conclusion}

Two anticoagulants were compared for perioperative analyses of platelet function in MEA. Impaired aggregometry was observed with both heparin and citrate. In contrast to citrated blood, MEA analyses performed in heparinized blood reflected intraoperative decrease and postoperative recovery of platelet aggregation. The choice of anticoagulant influences the measurements of platelet function and requires validation for different types of platelet analyzers. Further research is required to evaluate the clinical significance of the sample anticoagulant of platelet aggregometry in whole blood during different clinical indications.

\section{Disclosure}

The study was supported by the manufacturer of the presented method (Dynabyte Medical, Munich, Germany). Andreas Calatzis is coinventor of the presented method. None of the other aforementioned authors have any financial interest concerning the study methods.

\section{References}

Belcher PR, Muriithi EW, Milne EM, et al. 2000. Heparin, platelet aggregation, neutrophils, and cardiopulmonary bypass. Thromb Res, 98:249-56.

Boldt J, Knothe C, Zickmann B, et al. 1993. Comparison of two aprotinin dosage regimens in pediatric patients having cardiac operations. Influence on platelet function and blood loss. J Thorac Cardiovasc Surg, 105:705-11.

Boldt J, Schindler E, Osmer C, et al. 1994. Influence of different anticoagulation regimens on platelet function during cardiac surgery. $\mathrm{Br} J$ Anaesth, 73:639-44.

Born GV, Cross MJ. 1963. The aggregation of blood platelets. J Physiol, 168:178-95.

Bretschneider E, Glusa E, Schror K. 1994. ADP-, PAF- and adrenalineinduced platelet aggregation and thromboxane formation are not affected by a thromboxane receptor antagonist at physiological external Ca++ concentrations. Thromb Res, 75:233-42.

Cardinal DC, Flower RJ. 1980. The electronic aggregometer: a novel device for assessing platelet behavior in blood. J Pharmacol Methods, 3:135-58.

Chen JH, Karlberg KE, Sylvén C. 1991. Heparin and low molecular weight heparin but not hirudin stimulate platelet aggregation in whole blood from acetylsalicylic acid treated healthy volunteers. Thromb Res, 63:319-29. 
Glusa E, Markwardt F. 1990. Platelet functions in recombinant hirudinanticoagulated blood. Haemostasis, 20:112-8.

Hartmann M, Sucker C, Boehm O, et al. 2006. Effects of cardiac surgery on hemostasis. Transfus Med Rev, 20:230-41.

Kestin AS, Valeri CR, Khuri SF, et al. 1993. The platelet function defect of cardiopulmonary bypass. Blood, 82:107-17.

Kingston JK, Bayly WM, Sellon DC, et al. 2001. Effects of sodium citrate, low molecular weight heparin, and prostaglandin $\mathrm{E} 1$ on aggregation, fibrinogen binding, and enumeration of equine platelets. Am J Vet Res, 62:547-54.

Landesberg R, Burke A, Pinsky D, et al. 2005. Activation of platelet-rich plasma using thrombin receptor agonist peptide. J Oral Maxillofac Surg, 63:529-35.

Lavee J, Raviv Z, Smolinsky A, et al. 1993. Platelet protection by low-dose aprotinin in cardiopulmonary bypass: electron microscopic study. Ann Thorac Surg, 55:114-9.

Mengistu AM, Wolf MW, Boldt J, et al. 2008. Evaluation of a new platelet function analyzer in cardiac surgery: a comparison of modified thromboelastography and whole-blood aggregometry. J Cardiothorac Vasc Anesth, 22:40-6.

Muriithi EW, Belcher PR, Day SP, et al. 2000. Heparin-induced platelet dysfunction and cardiopulmonary bypass. Ann Thorac Surg, 69:1827-32.
Poston R, Gu J, Manchio J, et al. 2005. Platelet function tests predict bleeding and thrombotic events after off-pump coronary bypass grafting. Eur $J$ Cardiothorac Surg, 27:584-91.

Seyfert UT, Haubelt H, Vogt A, et al. 2007. Variables influencing Multiplate(TM) whole blood impedance platelet aggregometry and turbidimetric platelet aggregation in healthy individuals. Platelets, 18:199-206.

Slaughter TF, Sreeram G, Sharma AD, et al. Reversible shear-mediated platelet dysfunction during cardiac surgery as assessed by the PFA-100 platelet function analyzer. Blood Coagul Fibrinolysis, 12:85-93.

Tóth O, Calatzis A, Penz S, et al. 2006. Multiple electrode aggregometry: a new device to measure platelet aggregation in whole blood. Thromb Haemost, 96:781-8.

von Pape KW, Dzijan-Horn M, Bohner J, et al. 2007. [Control of aspirin effect in chronic cardiovascular patients using two whole blood platelet function assays. PFA-100 and Multiplate]. Hamostaseologie, 27:155-60.

Wallen NH, Ladjevardi M, Albert J, et al. 1997. Influence of different anticoagulants on platelet aggregation in whole blood; a comparison between citrate, low molecular mass heparin and hirudin. Thromb Res, 87:151-7. 